\title{
BAYESIAN LINEAR UNMIXING OF HYPERSPECTRAL IMAGES CORRUPTED BY COLORED GAUSSIAN NOISE WITH UNKNOWN COVARIANCE MATRIX
}

\author{
N. Dobigeon*, J.-Y. Tourneret* and A. O. Hero III' \\ * IRIT-ENSEEIHT, 2 rue Charles Camichel, BP 7122, 31071 Toulouse cedex 7, France \\ $\dagger$ University of Michigan, Department of EECS, Ann Arbor, MI 48109-2122, USA \\ \{nicolas.dobigeon,jean-yves.tourneret\}@enseeiht.fr, hero@eecs.umich.edu
}

\begin{abstract}
This paper addresses the problem of unmixing hyperspectral images contamined by additive colored noise. Each pixel of the image is modeled as a linear combination of pure materials (denoted as endmembers) corrupted by an additive zero mean Gaussian noise sequence with unknown covariance matrix. Appropriate priors are defined ensuring positivity and additivity constraints on the mixture coefficients (denoted as abundances). These coefficients as well as the noise covariance matrix are then estimated from their joint posterior distribution. A Gibbs sampling strategy generates abundances and noise covariance matrices distributed according to the joint posterior. These samples are then averaged for minimum mean square error estimation.
\end{abstract}

Index Terms - Bayesian inference, Monte Carlo methods, spectral unmixing, hyperspectral images.

\section{INTRODUCTION AND PROBLEM FORMULATION}

The spectral unmixing problem has received considerable attention in remote sensing, signal and image processing (see for instance [1] and references therein). Most spectral unmixing procedures assume that the observed spectrum of a mixed pixel is linearly mixed by a number of so-called endmembers with corresponding fractions referred to as abundances. More precisely, according to the linear mixing model (LMM) presented in [1], the $L$-spectrum $\mathbf{y}=$ $\left[y_{1}, \ldots, y_{L}\right]^{\top}$ of a mixed pixel is assumed to be a linear combination of $R$ spectra $\mathbf{m}_{r}$ corrupted by additive Gaussian noise:

$$
\mathbf{y}=\sum_{r=1}^{R} \mathbf{m}_{r} \alpha_{r}+\mathbf{n},
$$

where $\mathbf{m}_{r}=\left[m_{r, 1}, \ldots, m_{r, L}\right]^{\top}$ denotes the spectrum of the $r^{\text {th }}$ material, $\alpha_{r}$ is the fraction of the $r$ th material in the pixel, $R$ is the number of pure materials (or endmembers) present in all the observed scene and $L$ is the number of available spectral bands for the image. The $R$ endmembers spectra $\mathbf{m}_{r}$ are assumed to be known in this paper. In practical applications, they can be obtained by an endmember extraction procedure such as the well-known $\mathrm{N}$-finder (N-FINDR) algorithm developed by Winter [2]. Due to physical considerations, the fraction vector satisfies the following positivity and additivity constraints:

$$
\left\{\begin{array}{l}
\alpha_{r} \geq 0, \forall r=1, \ldots, R, \\
\sum_{r=1}^{R} \alpha_{r}=1
\end{array}\right.
$$

A classical assumption in hyperspectral imagery is that the noise vector $\mathbf{n}=\left[n_{1}, \ldots, n_{L}\right]^{\top}$ is white and Gaussian $[3,4]$. However, due to their intrinsic functioning, some actual spectrometers can provide hyperspectral images affected by colored noise [5].

This paper studies a new Bayesian linear unmixing algorithm for additive colored Gaussian noise. This algorithm allows one to analyze the impact of noise correlation on spectral unmixing. Appropriate prior distributions are chosen for the abundances to satisfy the positivity and additivity constraints, as in [6]. The noise vector $\mathbf{n}$ is assumed to be zero-mean Gaussian with covariance matrix $\boldsymbol{\Sigma}^{1}$ of dimension $L \times L$, denoted as $\mathbf{n} \sim \mathcal{N}\left(\mathbf{0}_{L}, \boldsymbol{\Sigma}\right)$ (where $\mathbf{0}_{L}$ is the vector made of $L$ zeros). A conjugate inverse Wishart distribution is then chosen for the covariance matrix $\boldsymbol{\Sigma}$. This prior reduces to a product of inverse gamma distributions when the noise sequence is independent and identically distributed (i.i.d.) as in [6]. The hyperparameters associated to the Bayesian unmixing model are assigned vague hyperpriors resulting in a hierarchical Bayesian model. The posterior distribution of the corresponding unknown model parameters is then derived. A Gibbs sampler is finally proposed to generate samples according to this posterior and to compute the minimum mean square error (MMSE) estimator of the unknown parameters.

The paper is organized as follows. The posterior distribution of the abundances and noise covariance matrix is derived in Section 2. Section 3 studies the Gibbs sampling strategy which generates samples distributed according this posterior. Simulation results are presented in Section 4. Conclusions are reported in Section 5.

\section{HIERARCHICAL BAYESIAN MODEL}

The likelihood and the priors inherent to the proposed hierarchical Bayesian model are defined for the spectral unmixing of hyperspectral images. A particular attention is devoted to defining abundance prior distributions satisfying positivity and additivity constraints. The noise correlation is also taken into account by specifying an inverse Wishart prior for the noise covariance matrix.

\subsection{Likelihood}

The linear mixing model defined in (1) and the statistical properties of the noise vector $\mathbf{n}$ lead to $\mathbf{y} \sim \mathcal{N}\left(\mathbf{M}^{+} \boldsymbol{\alpha}^{+}, \boldsymbol{\Sigma}\right)$, where $\mathbf{M}^{+}=$ $\left[\mathbf{m}_{1}, \ldots, \mathbf{m}_{R}\right]$ and $\boldsymbol{\alpha}^{+}=\left[\alpha_{1}, \ldots, \alpha_{R}\right]^{\top}$. As a consequence, the

\footnotetext{
${ }^{1}$ The covariance matrix of the colored noise should depend on $\mathbf{m}_{r}$ and $\alpha_{r}, r=1, \ldots, R$, in order to ensure positivity of the spectral components $y_{1}, \ldots, y_{L}$. However, choosing a noise covariance matrix independent of the endmembers and abundances provides a much simpler statistical model which is satisfactory as soon as $\sum_{r=1}^{R} \mathbf{m}_{r} \alpha_{r}$ is not too small (see $[1,3,4]$ for a similar assumption in the white noise case).
} 
likelihood function can be expressed as:

$$
f\left(\mathbf{y} \mid \boldsymbol{\alpha}^{+}, \boldsymbol{\Sigma}\right) \propto|\boldsymbol{\Sigma}|^{-\frac{1}{2}} \exp \left[-\frac{1}{2}\left\|\mathbf{y}-\mathbf{M}^{+} \boldsymbol{\alpha}^{+}\right\|_{\boldsymbol{\Sigma}^{-1}}^{2}\right]
$$

where $\propto$ means "proportional to", $|\cdot|$ stands for the determinant and $\|\cdot\|_{\mathbf{P}}$ is the Euclidian norm derived from the $\mathbf{P}$-scalar product $\langle\mathbf{x}, \mathbf{y}\rangle_{\mathbf{P}}=$ $\mathbf{x}^{\top} \mathbf{P} \mathbf{y}, \mathbf{P}$ being a $L \times L$ positive definite Hermitian matrix.

\subsection{Parameter priors}

\subsubsection{Abundance prior}

The abundance vector can be re-written as $\boldsymbol{\alpha}^{+}=\left[\boldsymbol{\alpha}^{\top}, \alpha_{R}\right]^{\top}$ with $\boldsymbol{\alpha}=\left[\alpha_{1}, \ldots, \alpha_{R-1}\right]^{\top}$ and $\alpha_{R}=1-\sum_{r=1}^{R-1} \alpha_{r}$. A uniform distribution on the simplex $\mathbb{S}$ is chosen as prior distribution for $\alpha$ :

$$
f(\boldsymbol{\alpha}) \propto \mathbf{1}_{\mathbb{S}}(\boldsymbol{\alpha}),
$$

where $\mathbf{1}_{\mathbb{S}}($.$) is the indicator function defined on$

$$
\mathbb{S}=\left\{\boldsymbol{\alpha} \mid \alpha_{r} \geq 0, \forall r=1, \ldots, R-1, \sum_{r=1}^{R-1} \alpha_{r} \leq 1\right\} .
$$

This prior ensures the abundances satisfy the positivity and additivity constraints and reflect the absence of additional knowledge regarding these parameters.

\subsubsection{Noise covariance matrix prior}

The prior distribution for the covariance matrix $\boldsymbol{\Sigma}$ is an inverse Wishart distribution with $\nu$ degrees of freedom and mean $\mathbf{D}$ :

$$
\boldsymbol{\Sigma} \mid \nu, \mathbf{D} \sim \mathcal{W}_{L}^{-1}((\nu-L-1) \mathbf{D}, \nu),
$$

where $\nu$ is a fixed hyperparameter ${ }^{2}$. The probability density function (pdf) of $\boldsymbol{\Sigma}$, conditionally to $\mathbf{D}$ can then be written [7]:

$$
f(\boldsymbol{\Sigma} \mid \nu, \mathbf{D})=\frac{(\nu-L-1)^{\frac{\nu}{2}}|\mathbf{D}|^{\frac{\nu}{2}}}{c(L, \nu)|\boldsymbol{\Sigma}|^{\frac{(\nu+L+1)}{2}}} \operatorname{etr}\left\{-\frac{1}{2}(\nu-L-1) \boldsymbol{\Sigma}^{-1} \mathbf{D}\right\},
$$

where $|$.$| and \operatorname{etr}\{$.$\} stand for the determinant and the exponential of$ the trace of the matrix between braces, and $c(L, \nu)$ is a normalization constant:

$$
c(L, \nu)=2^{\frac{L \nu}{2}} \pi^{\frac{L(L-1)}{4}} \prod_{i=1}^{L} \Gamma\left(\frac{\nu-L+i}{2}\right),
$$

where $\Gamma(\cdot)$ denotes the Gamma function. The inverse Wishart distribution is a conjugate prior for $\boldsymbol{\Sigma}$. The parameter $\nu$ allows one to adjust the amount of prior knowledge we have regarding $\boldsymbol{\Sigma}$. Indeed, straightforward computations lead to:

$$
\operatorname{var}[\boldsymbol{\Sigma} \mid \nu, \mathbf{D}]=\frac{(\nu-L+1) \mathbf{D}^{2}+(\nu-L-1) \operatorname{tr}\{\mathbf{D}\} \mathbf{D}}{(\nu-L-3)(\nu-L)},
$$

which approaches 0 when $\nu$ increases. Therefore, the distance between $\boldsymbol{\Sigma}$ and $\mathbf{D}$ is directly related to the value of $\nu$. Moreover, the chosen inverse Wishart distribution ensures that the mean of $\boldsymbol{\Sigma}$ is $D$. In the context of linear spectral unmixing, we propose to consider a

\footnotetext{
${ }^{2}$ The hyperparameter $\nu$ has to satisfy the condition $\nu>L+3$ in order to ensure the existence of the mean and variance of the inverse Wishart distribution.
}

diagonal hyperparameter matrix $\mathbf{D}=\gamma \mathbf{I}_{L}$, where $\gamma$ is a positive adjustable hyperparameter. This particular choice reflects the fact that the noise variances change from one spectral band to another but not significantly ${ }^{3}$. In this case, the noise covariance matrix prior can be written:

$$
f(\boldsymbol{\Sigma} \mid \nu, \gamma) \propto \frac{\gamma^{\frac{\nu L}{2}}}{|\boldsymbol{\Sigma}|^{\frac{(\nu+L+1)}{2}}} \operatorname{etr}\left\{-\frac{\gamma}{2}(\nu-L-1) \boldsymbol{\Sigma}^{-1}\right\} .
$$

\subsection{Hyperparameter priors}

The prior of $\gamma$ is a non-informative Jeffrey's prior:

$$
f(\gamma) \propto \frac{1}{\gamma} \mathbf{1}_{\mathbb{R}^{+}}(\gamma)
$$

This prior reflects the lack of knowledge regarding the hyperparameter $\gamma$. The hyperparameter $\nu$ which adjusts the distance between $\boldsymbol{\Sigma}$ and $D$ (or equivalently the amount of available prior information) is fixed in the proposed implementation because this choice has not appeared to be critical in our simulations. However, a modified hierarchical algorithm allowing one to estimate $\nu$ could also be developed.

\subsection{Posterior distribution of $\theta$}

The posterior distribution of the unknown parameter vector $\boldsymbol{\theta}=$ $\{\boldsymbol{\alpha}, \boldsymbol{\Sigma}\}$ can be computed from the following hierarchical structure:

$$
f(\boldsymbol{\theta} \mid \mathbf{y})=\int f(\boldsymbol{\theta}, \gamma \mid \mathbf{y}) d \gamma \propto \int f(\mathbf{y} \mid \boldsymbol{\theta}) f(\boldsymbol{\theta} \mid \gamma) f(\gamma) d \gamma
$$

where $f(\mathbf{y} \mid \boldsymbol{\theta})$ and $f(\gamma)$ have been defined in Eq.'s (3) and (11). Moreover, by assuming a priori independence between $\boldsymbol{\Sigma}$ and $\boldsymbol{\alpha}$, the following result can be obtained:

$$
f(\boldsymbol{\theta} \mid \gamma)=f(\boldsymbol{\alpha}) f(\boldsymbol{\Sigma} \mid \nu, \gamma) .
$$

This hierarchical structure allows one to integrate out the hyperparameter $\gamma$ from the joint distribution $f(\boldsymbol{\theta}, \gamma \mid \mathbf{y})$, yielding:

$$
\begin{aligned}
f(\boldsymbol{\theta} \mid \mathbf{y}) & \propto\left(\operatorname{tr}\left[\Sigma^{-1}\right]\right)^{-\frac{\nu L}{2}}|\boldsymbol{\Sigma}|^{-\frac{\nu+L+1}{2}} \\
& \times \exp \left[-\frac{1}{2}\left\|\mathbf{y}-\mathbf{M}^{+} \boldsymbol{\alpha}^{+}\right\|_{\boldsymbol{\Sigma}^{-1}}^{2}\right] \mathbf{1}_{\mathbb{S}}(\boldsymbol{\alpha}),
\end{aligned}
$$

where $\operatorname{tr}\{\cdot\}$ is the trace operator. Of course, this posterior distribution it too complicated to derive the MMSE or the maximum a posteriori estimator of $\theta$. The next section studies a Gibbs sampling strategy which generates abundances and noise covariance matrices distributed according to the full posterior (14).

\section{GIBBS SAMPLER}

The Gibbs sampler generates samples distributed according to the full conditional distributions, i.e. alternatively according to $f(\boldsymbol{\alpha} \mid \boldsymbol{\Sigma}, \mathbf{y})$ and $f(\boldsymbol{\Sigma} \mid \boldsymbol{\alpha}, \mathbf{y})$, as detailed below.

\subsection{Generation of samples distributed according to $f(\boldsymbol{\alpha} \mid \boldsymbol{\Sigma}, \mathbf{y})$}

By denoting $\mathbf{M}=\left[\mathbf{m}_{1}, \ldots \mathbf{m}_{R-1}\right]$, straightforward computations yield:

$$
f(\boldsymbol{\alpha} \mid \boldsymbol{\Sigma}, \mathbf{y}) \propto \exp \left[-\frac{(\boldsymbol{\alpha}-\boldsymbol{\mu})^{\top} \boldsymbol{\Lambda}^{-1}(\boldsymbol{\alpha}-\boldsymbol{\mu})}{2}\right] \mathbf{1}_{\mathbb{S}(\boldsymbol{\alpha}),}
$$

${ }^{3} \mathrm{~A}$ more general algorithm could be derived by assuming $\mathbf{D}$ is any diagonal matrix. However, the proposed strategy with $\mathbf{D}=\gamma \mathbf{I}_{L}$ is a direct generalization of the algorithm derived in [6] for colored noise. 
where

$$
\left\{\begin{array}{l}
\boldsymbol{\Lambda}=\left[\left(\mathbf{M}-\mathbf{m}_{R} \mathbf{u}^{\top}\right)^{\top} \boldsymbol{\Sigma}^{-1}\left(\mathbf{M}-\mathbf{m}_{R} \mathbf{u}^{\top}\right)\right]^{-1}, \\
\boldsymbol{\mu}=\boldsymbol{\Lambda}\left[\left(\mathbf{M}-\mathbf{m}_{R} \mathbf{u}^{\top}\right)^{\top} \boldsymbol{\Sigma}^{-1}\left(\mathbf{y}-\mathbf{m}_{R}\right)\right]
\end{array}\right.
$$

with $\mathbf{u}=[1, \ldots, 1]^{\top} \in \mathbb{R}^{R-1}$. As a consequence, $\boldsymbol{\alpha} \mid \boldsymbol{\Sigma}, \mathbf{y}$ is distributed according to the following truncated Gaussian distribution:

$$
\boldsymbol{\alpha} \mid \boldsymbol{\Sigma}^{-1}, \mathbf{y} \sim \mathcal{N}_{\mathbb{S}}(\boldsymbol{\mu}, \boldsymbol{\Lambda}) .
$$

Note that this conditional distribution is concentrated on the simplex $\mathbb{S}$. Consequently, the generated abundances satisfy the positivity and additivity constraints, as requested.

\subsection{Generation of samples distributed according to $f(\Sigma \mid \boldsymbol{\alpha}, \mathbf{y})$}

To sample according to $f(\boldsymbol{\Sigma} \mid \boldsymbol{\alpha}, \mathbf{y})$, it is very convenient to generate samples distributed according to the joint distribution $f(\boldsymbol{\Sigma}, \gamma \mid \boldsymbol{\alpha}, \mathbf{y})$ by using the following two-step procedure:

\subsubsection{Generation of samples according to $f(\gamma \mid \boldsymbol{\Sigma}, \boldsymbol{\alpha}, \mathbf{y})$}

The posterior distribution of the hyperparameter $\gamma$ is:

$$
\gamma \mid \boldsymbol{\alpha}, \boldsymbol{\Sigma}, \mathbf{y} \sim \mathcal{G}\left(\frac{\nu L}{2}, \frac{\nu-L-1}{2} \operatorname{tr}\left\{\boldsymbol{\Sigma}^{-1}\right\}\right),
$$

where $\mathcal{G}(a, b)$ is the Gamma distribution with parameters $a$ and $b$.

\subsubsection{Generation of samples according to $f(\boldsymbol{\Sigma} \mid \gamma, \boldsymbol{\alpha}, \mathbf{y})$}

Straightforward computations show that the posterior distribution of $\boldsymbol{\Sigma}$ is an inverse Wishart distribution:

$$
\boldsymbol{\Sigma} \mid \gamma, \boldsymbol{\alpha}, \mathbf{y} \sim \mathcal{W}_{L}^{-1}\left(\gamma(\nu-L-1) \mathbf{I}_{L}+\mathbf{z z}^{\top}, \nu+1\right),
$$

where $\mathbf{z}=\mathbf{y}-\mathbf{M}^{+} \boldsymbol{\alpha}^{+}$.

The final Gibbs sampler is summarized in the algorithm below:

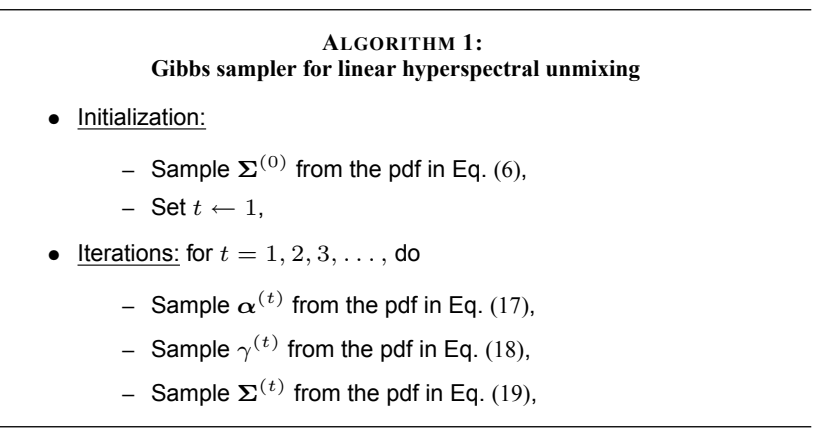

\section{SIMULATIONS}

This section illustrates the performance of the proposed abundance estimation procedure in the presence of additive colored Gaussian noise. For this, we consider a synthetic pixel resulting from the combination of three endmembers representative of a urban or suburban environment: green grass, bare red brick and galvanized steel metal. These endmembers have been extracted from the spectral libraries distributed with the ENVI package [8, p. 1035]. The proportions of these components (i.e. abundances) are $\alpha_{1}=0.05, \alpha_{2}=0.6$ and $\alpha_{3}=0.35$, respectively. The number of spectral bands is $L=413$. The observations have been corrupted by an additive zero-mean colored Gaussian noise $\mathbf{n}$ with covariance matrix $\boldsymbol{\Sigma}$. The $L \times L$ definite positive matrix $\boldsymbol{\Sigma}$ has been randomly drawn from an inverse Wishart distribution $\mathcal{W}_{L}^{-1}(\nu,(\nu-L-1) \mathbf{D})$ with mean $\mathbf{D}=\gamma \mathbf{I}_{L}$. All simulations presented in this section have been obtained with $\gamma=4.8 \times 10^{-3}$. This value of $\gamma$ corresponds to an average SNR:

$$
\mathrm{ASNR}=\frac{1}{\gamma L}\left\|\mathbf{M}^{+} \boldsymbol{\alpha}^{+}\right\|^{2}=15 \mathrm{~dB} .
$$

The degree of freedom $\nu$ of the inverse Wishart distribution has been chosen as $\nu=L+3+\eta, \eta \in \mathbb{N}^{*}$, in order to ensure the existence of the mean and variance of the noise covariance matrix prior. To understand the physical significance of the parameter $\eta$, consider the limit case $\eta \gg 1$. For large values of $\eta$, the variance of the Wishart distribution defined in (9) approaches 0 . As a consequence, the noise vector $\mathbf{n}$ reduces to an i.i.d. zero-mean Gaussian sequence with variance $\gamma$. The $50 \times 50$ first elements of the covariance matrix $\boldsymbol{\Sigma}$ are represented in Fig. 1 for different values of the parameter $\eta$ (the largest coefficients of $\boldsymbol{\Sigma}$ appear in white color). It can be clearly seen that the matrix $\boldsymbol{\Sigma}$ tends to be diagonal when $\eta$ increases. The parameter $\eta$ has been fixed to $\eta=30$ in our simulations.
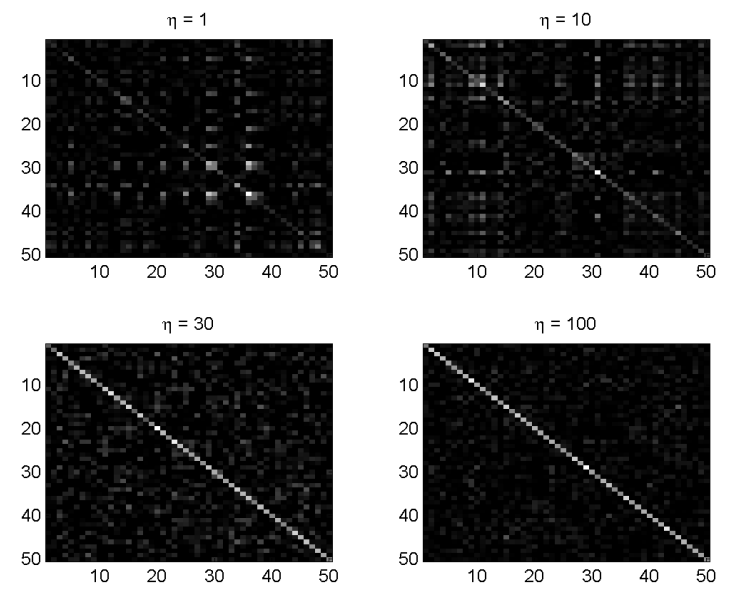

Fig. 1. The first $50 \times 50$ coefficients of the covariance matrix $\boldsymbol{\Sigma}$ for different values of $\eta\left(\gamma=4.8 \times 10^{-3}\right)$.

The next figures show the performance of the proposed unmixing algorithm. The endmember spectra corresponding to green grass, bare red brick and galvanized steel metal, as well as the resulting spectrum of the mixed pixel are depicted in Fig. 2. Figure 3 shows the posterior distributions of the abundances $\alpha_{r}(r=1,2,3)$ obtained for $N_{\mathrm{MC}}=30000$ iterations (including $N_{\mathrm{bi}}=10000$ burn-in iterations). These distributions are in good agreement with the actual values of $\boldsymbol{\alpha}^{+}=[0.05,0.6,0.35]^{\mathrm{T}}$. As a comparison, the posterior distributions of the abundance coefficients estimated by the unmixing procedure of [6] assuming that the noise sequence is i.i.d. are depicted in Fig. 3 (dotted lines). The variances of the estimated abundances are clearly smaller when the noise correlation is taken into account. This result is confirmed in Table 1 which reports the means and variances of the abundance estimates for the two algorithms (computed from 50 Monte Carlo runs). 

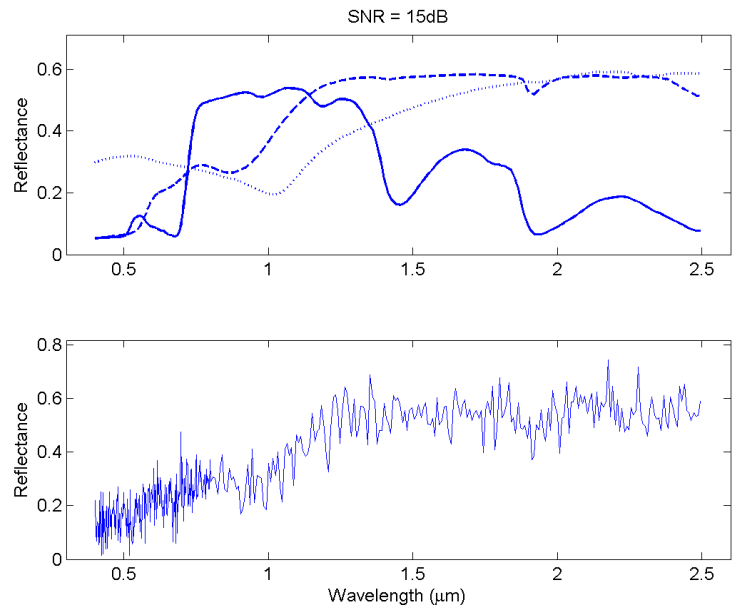

Fig. 2. Top: endmember spectra: green grass (solid line), bare red brick (dashed line), galvanized steel metal (dotted line). Bottom: resulting spectrum of the mixed pixel.

It is interesting to note that the proposed algorithm generates samples distributed according to the posterior distribution of the covariance matrix $\boldsymbol{\Sigma}$. These samples can be used to extract useful information regarding the noise affecting hyperspectral images. As an example, the posteriors of two diagonal elements of $\boldsymbol{\Sigma}$ are depicted in Fig. 4, illustrating that the noise correlations are small compared to their variances.


Fig. 3. Posterior distributions of the abundances $\left[\alpha_{1}, \alpha_{2}, \alpha_{3}\right]^{\top}$ estimated by the proposed algorithm (continuous lines) and by the algorithm in [6] (dotted lines).

\section{CONCLUSIONS}

A new hierarchical Bayesian unmixing algorithm was derived for hyperspectral images corrupted by additive colored Gaussian noise. The results obtained on synthetic signals showed that assuming the noise is i.i.d. can result in poor abundance estimations if the noise
Table 1. Means and variances of the abundance estimates computed from 50 Monte Carlo runs.

\begin{tabular}{|c|c|c|}
\cline { 2 - 3 } \multicolumn{1}{c|}{} & Proposed approach & Approach in [6] \\
\hline \hline $\mathrm{E}\left[\widehat{\alpha_{1}}\right]$ & 0.050 & 0.052 \\
$\mathrm{E}\left[\widehat{\alpha_{2}}\right]$ & 0.600 & 0.594 \\
$\mathrm{E}\left[\widehat{\alpha_{2}}\right]$ & 0.350 & 0.354 \\
\hline \hline $\operatorname{var}\left[\widehat{\alpha_{1}}\right]$ & $1.8 \times 10^{-4}$ & $5.9 \times 10^{-4}$ \\
$\operatorname{var}\left[\widehat{\alpha_{2}}\right]$ & $7.4 \times 10^{-4}$ & $2.8 \times 10^{-3}$ \\
$\operatorname{var}\left[\widehat{\alpha_{2}}\right]$ & $5.5 \times 10^{-4}$ & $2.2 \times 10^{-3}$ \\
\hline \hline
\end{tabular}
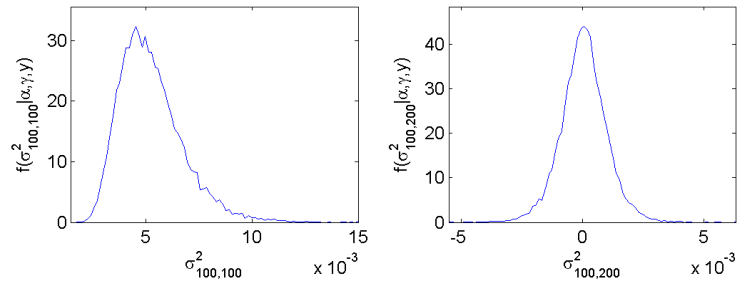

Fig. 4. Posterior distributions of typical diagonal and off-diagonal elements of $\boldsymbol{\Sigma}$.

is correlated. Future investigations include the study of unmixing algorithms for the joint estimation of endmembers and abundances. It would also be interesting to generalize the proposed approach to more sophisticated noise models described by statistical mixtures [9] or sums of several multivariate normal probability distributions [10].

\section{REFERENCES}

[1] N. Keshava and J. F. Mustard, "Spectral unmixing," IEEE Signal Processing Magazine, pp. 44-57, Jan. 2002.

[2] M. E. Winter, "Fast autonomous spectral end-member determination in hyperspectral data," in Proc. 13th Int. Conf. on Applied Geologic Remote Sensing, vol. 2, Vancouver, April 1999, pp. 337-344.

[3] C.-I Chang, X.-L. Zhao, M. L. G. Althouse, and J. J. Pan, "Least squares subspace projection approach to mixed pixel classification for hyperspectral images," IEEE Trans. Geosci. and Remote Sensing, vol. 36, no. 3, pp. 898-912, May 1998.

[4] D. Manolakis, C. Siracusa, and G. Shaw, "Hyperspectral subpixel target detection using the linear mixing model," IEEE Trans. Geosci. and Remote Sensing, vol. 39, no. 7, pp. 1392-1409, July 2001.

[5] C.-I Chang and Q. Du, "Estimation of number of spectrally distinct signal sources in hyperspectral imagery," IEEE Trans. Geosci. and Remote Sensing, vol. 42, no. 3, pp. 608-619, March 2004.

[6] N. Dobigeon and J.-Y. Tourneret, "Spectral unmixing of hyperspectral images using a hierarchical Bayesian model," in Proc. IEEE Int. Conf. Acoust., Speech, and Signal Processing (ICASSP), vol. 3, Honolulu, Hawaii, USA, April 2007, pp. 1209-1212.

[7] J. A. Tague and C. I. Caldwell, "Expectations of useful complex Wishart forms," Multidimensional Systems and Signal Processing, vol. 5, no. 3, pp. 263-279, July 2004.

[8] RSI (Research Systems Inc.), ENVI User's guide Version 4.0, Boulder, CO 80301 USA, Sept. 2003.

[9] C. J. Willis, "Mixture models for anomaly detection in hyperspectral imagery," in Military Remote Sensing, G. W. Kamerman and D. V. Willetts, Eds., vol. 5613, no. 1. SPIE, Dec. 2004, pp. 119-128.

[10] I. Kasen, P. E. Goa, and T. Skauli, "Target detection in hyperspectral images based on multi-component statistical models for representation of background clutter," in Electro-Optical and Infrared Systems: Technology and Applications, R. G. Driggers and D. A. Huckridge, Eds., vol. 5612, no. 1. SPIE, Dec. 2004, pp. 258-264. 